\title{
Impact of a minority relativistic electron tail interacting with a thermal plasma containing high-atomic-number impurities
}

Nathan A. Garland, ${ }^{1, a)}$ Hyun-Kyung Chung, ${ }^{2}$ Christopher J. Fontes, ${ }^{1}$ Mark C. Zammit, ${ }^{1}$ James Colgan, ${ }^{1}$ Todd Elder, ${ }^{1,3}$ Christopher J. McDevitt, ${ }^{1,4}$ Timothy M. Wildey, ${ }^{5}$ and Xian-Zhu Tang ${ }^{1}$

${ }^{1)}$ Los Alamos National Laboratory, Los Alamos, NM 87545, USA

2) National Fusion Research Institute (NFRI), 169-148 Gwahak-ro, Yuseong-gu, Daejeon 34133,

Korea

${ }^{3)}$ Columbia University, New York, NY 10027, USA

4) Department of Materials Science and Engineering, University of Florida, Gainesville, FL 32611,

USA

${ }^{5)}$ Sandia National Laboratories, Albuquerque, NM 87185, USA

(Dated: 6 April 2020)

A minority relativistic electron component can arise in both laboratory and naturally-occurring plasmas. In the presence of high-atomic-number ion species, the ion charge state distribution at low bulk electron temperature can be dominated by relativistic electrons, even though their density is orders of magnitude lower. This is due to the relativistic enhancement of the collisional excitation and ionization cross sections. The resulting charge state effect can dramatically impact the radiative power loss rate and the related Bethe stopping power of relativistic electrons in a dilute plasma.

Major disruptions must be adequately mitigated in a tokamak for a viable fusion power reactor, with the current approach for ITER's Disruption Mitigation System (DMS) based on injecting large quantities of high-atomic-number impurities such as argon or neon ${ }^{1,2}$. This can serve the dual purpose of thermal quench mitigation by spreading the plasma power load over the reactor first wall through radiative cooling of the bulk plasma ${ }^{3-5}$, and current quench mitigation via enhanced runaway electron current dissipation due to increased collision rates ${ }^{4,6,7}$. The resulting plasma mixture of hydrogenic fuel, helium ash, and high-Z impurities, at a total atomic number density of $10^{20-21} \mathrm{~m}^{-3}$, leads to a radiatively cooled bulk electron population, along with a minority runaway electron component at a number density of $10^{16-17} \mathrm{~m}^{-3}$, which is sufficient to carry a plasma current of a few to 10 megaamperes (MA) in ITER discharges. This is a most unusual plasma that emphasizes a variety of radiative processes for plasma power loss, some combination of which point to the surprising role of minority relativistic runaway electrons. Although the presentation here focuses on the conditions of a disrupting tokamak plasma, the issues uncovered apply to a range of plasma applications in which a minority population of relativistic electrons co-exists with a cold thermal bulk that contains high-atomic-number ion species. Some non-fusion examples include atmospheric lightning ${ }^{8}$, planetary radiation belts ${ }^{9}$, and any synchrotron emitting astrophysical plasma that is also metal rich $^{10}$

In a mitigated tokamak post-disruption discharge where $n_{\mathrm{Ar}}=n_{\mathrm{D}}=10^{20} \mathrm{~m}^{-3}$, line radiation by argon would dominate the plasma radiative power loss, which is around a few $\mathrm{GW} / \mathrm{m}^{3}$ over the electron temperature range of $10 \mathrm{eV}$ to $1 \mathrm{keV}$. For a post-thermal quench ITER plasma of $300 \mathrm{~m}^{3}$ in volume and $300 \mathrm{MJ}$ in thermal energy, this loss mechanism alone would ensure a thermal collapse (electron temperature, $T_{e}$, from a few $\mathrm{keV}$ to below $10 \mathrm{eV}$ ) over $1 \mathrm{~ms}$ or less. Once

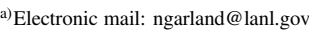

the electron thermal temperature reaches a few $\mathrm{eV}$, radiative loss in a thermal plasma of comparable argon and deuterium number density becomes negligibly inefficient. The minority runaway electron population, despite the tiny number density of $10^{16-17} \mathrm{~m}^{-3}$ required to carry a few to $10 \mathrm{MA}$ of current in ITER, would dominate the radiative loss. The underlying cause for runaway electrons aiding efficient radiative power loss is fundamentally due to a quantum electrodynamics (QED) effect dating back to Møller, Breit, and Bethe.

At electron energies significantly above the threshold energy, the excitation and ionization integrated cross-section (ICS) would normally decrease with increasing impacting electron energy. However, this trend reverses when the impacting electron reaches relativistic energies, usually at a threshold around one MeV. This is a lowest-order QED correction associated with the Møller or generalized Breit interaction $^{11-15}$. Much of these physical effects are captured by the Bethe formula for inelastic electron stopping ${ }^{16-19}$

$$
\begin{array}{r}
-\frac{d E}{d x}=\sum_{\alpha} \frac{e^{4} n_{e \alpha}}{4 \pi m_{e} v^{2} \varepsilon_{0}^{2}}\left[\ln \left(\frac{m_{e}^{2} c^{4}\left(\gamma^{2}-1\right)(\gamma-1)}{2\left\langle I_{\alpha}\right\rangle^{2}}\right)\right. \\
\left.-\left(\frac{2}{\gamma}-\frac{1}{\gamma^{2}}\right) \ln 2+\frac{1}{\gamma^{2}}+\frac{(\gamma-1)^{2}}{8 \gamma^{2}}\right],
\end{array}
$$

where $v$ is the electron speed, $\gamma$ is the relativistic Lorentz factor, $c$ is the speed of light, $\varepsilon_{0}$ the vacuum permittivity, and $e$ and $m_{e}$ are the electron charge and rest mass respectively. For each ion species in a specific charge state, which is denoted by subscript $\alpha$, there is a unique mean excitation energy $\left\langle I_{\alpha}\right\rangle$ and bound electron density $n_{e \alpha}$. In fact, the standard treatment in runaway modeling is to incorporate the runaway slowing down due to excitation and ionization via a friction in the Fokker-Planck collision operator using the Bethe formula $^{20,21}$. The effect of ion charge state distribution (CSD) on runaway and thermal bulk plasma evolution is through a collisional-radiative (CR) model that deals with only the background Maxwellian population at given temperature.

In a dilute fusion plasma, such as those in tokamak disrup- 
tion, the aforementioned decoupled treatment of background thermal electrons and runaways ${ }^{20,21}$ has a straightforward prediction for the radiative power loss as the bulk electrons are cooled to below a few eV. Namely, the background electron density drops precipitously due to cold thermal electron recombination with ions. Along with a mean charge number $\langle Z\rangle$ approaching zero, the radiative power loss by thermal electrons can decrease by 5 orders of magnitude between $T_{e}=5 \mathrm{eV}$ to $T_{e}=1 \mathrm{eV}$. Meanwhile, as $\langle Z\rangle \rightarrow 0$ with $T_{e} \rightarrow 0$ the simultaneous increase in bound electron density $n_{e \alpha}$ and decrease in mean excitation potential $\left\langle I_{\alpha}\right\rangle$ leads to enhanced radiative loss by runaways, according to the Bethe formula.

Unlike in a solid, inelastic collisions between runaways and high-atomic-number impurities in a dilute plasma can directly alter the charge state of the plasma ions. The change in ion CSD, in turn, modifies both the radiative power loss by thermal electrons and the runaways. The former is through modified line emissions as the dominant bound-bound transitions have explicit charge state dependence, while the latter, using the Bethe stopping power formula, is by way of modified bound electron density $n_{e \alpha}$ and mean excitation potential $\left\langle I_{\alpha}\right\rangle$. This Letter provides $C R$ modeling taking into account both the thermal bulk and the runaways with enhanced excitation and ionization scattering processes, which allows us to not only elucidate the new qualitative trends enabled by the coupling between the minority runaways and bulk thermal population through high-atomic-number ions, but also quantify the discrepancies between a full CR treatment and a decoupled treatment of thermal bulk and minority runaways, which has been the state of the art in runaway modeling.

Our CR model is a fork (called FLYCHKLite) of the FLYCHK model/code, which is widely used in dense plasma applications ${ }^{23}$, for its demonstrated applicability of a screened hydrogenic super-configuration model to sufficiently replicate the required physics in a computationally efficient manner ${ }^{24,25}$. The Los Alamos suite of atomic physics codes ${ }^{12}$ have been employed to calibrate and verify the model, especially the contribution from $\Delta n=0$ transitions in line emissions. The robustness of our physics findings is also assessed via an uncertainty quantification analysis on the relativistic ICS for both excitation and ionization. We note that both FLY$\mathrm{CHK}^{23}$ and $\mathrm{ATOMIC}^{12}$ treat Auger processes by including multiply-excited states and inner-shell excited states. More exotic multi-Auger events ${ }^{41}$, such as "shake off," are not included in either codes. Their correction to single ionization cross section is a few percent for Ar.

Relativistic inelastic scattering: To account for the collisional processes of an arbitrary electron energy distribution function (EEDF), we need the ICS for electron impact excitation and ionization, in both the non-relativistic and relativistic energy regimes. Our approach is to employ non-relativistic analytic fits used in FLYCHK, augmented by the addition of an analytic relativistic correction following a Møller-Bethelike functional form ${ }^{13,15,26}$. For electron-impact excitation, the analytic form of van Regemorter ${ }^{27}$ is used for the non- relativistic regime,

$$
\sigma_{i \rightarrow j}^{\mathrm{NR}}=\frac{8 \pi^{2} a_{0}^{2}}{\sqrt{3}}\left(\frac{R y}{\Delta E_{i j}}\right)^{2} \frac{f_{i j} g(U)}{U},
$$

where $U=E / \Delta E_{i j}$ is the scaled incoming electron energy, $R y$ is the Rydberg energy, $f_{i j}$ is the collision oscillator strength and $a_{0}$ is the Bohr radius. The Gaunt factor is

$$
g(U)=A \log U+B+C(U+a)^{-1}+D(U+a)^{-2},
$$

where coefficients are found via empirical fits of (3) to averaged hydrogenic ICS computed by Chung et al. ${ }^{28}$ via planewave Born calculations augmented by near-threshold scaling of $\mathrm{Kim}^{29}$.

Using (2) as a basis, a Møller-Bethe-like relativistic correction $^{16,26,30}$ is applied so the total ICS is

$$
\sigma_{i \rightarrow j}^{\mathrm{TOT}}=(1-S(E)) \sigma_{i \rightarrow j}^{\mathrm{NR}}+S(E) \sigma_{i \rightarrow j}^{\mathrm{R}},
$$

where

$$
\sigma_{i \rightarrow j}^{\mathrm{R}}=\frac{8 \pi a_{0}^{2} R y^{2}}{m_{e} c^{2} \beta^{2}} \frac{f_{i j}}{\Delta E_{i j}}\left[\log \left(\frac{\beta^{2}}{1-\beta^{2}} \frac{m_{e} c^{2}}{2 \Delta E_{i j}}\right)-\beta^{2}\right],
$$

and the sigmoid smoothing function is defined as

$$
S(E)=\left[1+\exp \left(10^{-5}\left(10^{5}-E\right)\right)\right]^{-1},
$$

with $E$ in units of $\mathrm{eV}$. The form of (6) is chosen to adequately describe known relativistic ICS for inelastic processes.

For electron impact ionization, the non-relativistic ICS is modeled by the semi-empirical expression of Burgess and Chidichimo $^{31}$

$$
\sigma_{(Z, i) \rightarrow(Z+1, j)}^{\mathrm{NR}}=\pi a_{0}^{2} C \xi\left(\frac{R y}{\Delta I_{Z}^{i}}\right)^{2} \frac{1}{U} \log (U) W(U),
$$

where $\Delta I_{Z}^{i}$ is the ionization threshold energy for the level $i$ of charge state $Z, U=E / \Delta I_{Z}^{i}, \xi$ is the number of electrons in the shell being ionized, $C$ is an arbitrary constant nominally around $2^{31}$, and

$$
\begin{array}{r}
W(U)=(\log (U))^{\frac{\beta^{*}}{U},} \\
\beta^{*}=0.25 \sqrt{\frac{100 Q_{n}+91}{4 Q_{n}+3}}-1.25,
\end{array}
$$

where $Q_{n}$ is the screened charge ${ }^{23}$.

Again using the same form as (4) for the total ionization ICS, we add a relativistic correction ${ }^{13,32}$ on top of (7) following the form

$$
\begin{aligned}
\sigma_{(Z, i) \rightarrow(Z+1, j)}^{\mathrm{R}}= & \pi a_{0}^{2} C \xi\left(\frac{R y}{\Delta I_{Z}^{i}}\right) \\
& {\left[\log \left(\frac{\beta^{2}}{1-\beta^{2}} \frac{m_{e} c^{2}}{2 \Delta I_{Z}^{i}}\right)-\beta^{2}\right] . }
\end{aligned}
$$

Figs. 1 and 2 compare the proposed fits with calculated ICS for excitation and ionization in the literature. We note agreement within a factor of two of recent R-matrix theories at the 


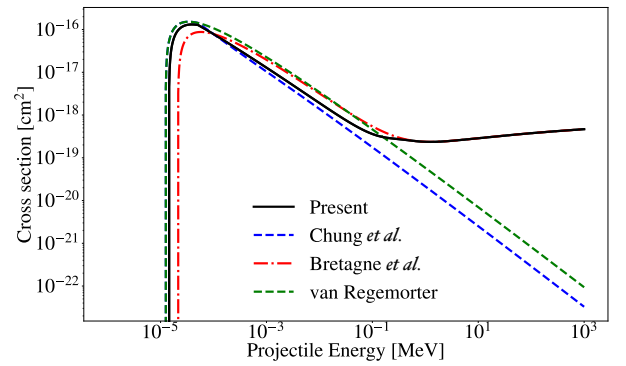

FIG. 1. ICS for excitation of Cl-like $\mathrm{Ar}^{+} n=3 \rightarrow n=4$ transition. Comparison of our proposed approximate ICS is made against the non-relativistic results of Chung et al. ${ }^{28}$, van Regemorter ${ }^{27}$ and the relativistic result of Bretagne et al. ${ }^{26}$.

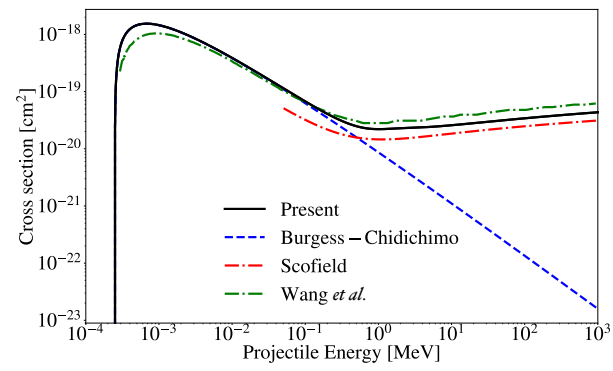

FIG. 2. ICS for lumped-average L-shell ionization of Ar. Comparison of our proposed approximate ICS is made against the nonrelativistic result of Burgess and Chidichimo ${ }^{31}$, and the relativistic results of Scofield ${ }^{13}$ and Wang et al. ${ }^{33}$.

peak of the cross section, and that unlike those reported in Ref. ${ }^{33}$, normally such calculations are not extended into the relativistic regime of relevance here. The plasma physics impact established in this paper certainly points to the importance and urgency of such systematic first-principle cross section calculations.

Simulation problem setup: To demonstrate the impact of using ICS that describe enhanced scattering observed at relativistic energies we perform a comparison between CR model results computed with and without relativistic ICS effects applied to conditions representative of a tokamak disruption mitigation scenario. Without losing generality, the electron distribution model is defined as a bulk Maxwellian at given temperature $T_{e}$ and a Gaussian runaway tail that has a mean energy $\left\langle E_{0}\right\rangle$ and full width at half maximum spread of $\Delta E_{\mathrm{FWHM}}$ at runaway number density $n_{\mathrm{RE}}$. The ion population is fixed with $\left(n_{\mathrm{D}}, n_{\mathrm{Ar}}\right)$. The thermal bulk then has an electron density $\left(n_{e}\right)$ from the quasi-neutral condition $n_{e}=\langle Z\rangle_{\mathrm{Ar}} n_{\mathrm{Ar}}+$ $\langle Z\rangle_{\mathrm{D}} n_{\mathrm{D}}-n_{\mathrm{RE}}$. As $T_{e}$ varies from $10 \mathrm{keV}$ to $1 \mathrm{eV}, n_{e}$ can

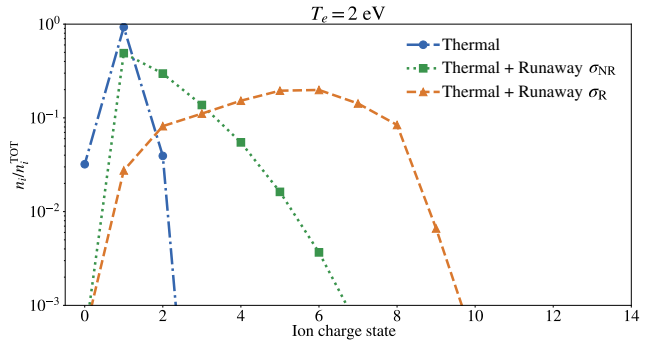

FIG. 3. Argon ion CSD at $T_{e}=2 \mathrm{eV}$ with a relativistic Gaussian tail supporting a $10 \mathrm{MA}$ current compared to a thermal discharge. The impact of including relativistic ICS effects $(\boldsymbol{\Lambda})$ compared to using ICS without the Møller/generalized Breit interaction ( $\mathbf{\square}$ ) or simply assuming a thermal plasma $(\bullet)$ is apparent.

vary greatly as a function of $\langle Z\rangle$, but $n_{\mathrm{RE}}$ is a fixed number that only depends on how much current the runaways need to carry. For example, to carry $10 \mathrm{MA}$ of runaway current in ITER, $n_{\mathrm{RE}} \approx 10^{17} \mathrm{~m}^{-3}$. Our scan over $T_{e}$ is performed with fixed $\left\langle E_{0}\right\rangle=10 \mathrm{MeV}, \Delta E_{\mathrm{FWHM}}=5 \mathrm{MeV}, n_{\mathrm{RE}}=10^{17} \mathrm{~m}^{-3}$, and $n_{\mathrm{D}}=n_{\mathrm{Ar}}=10^{20} \mathrm{~m}^{-3}$. The CR model iteratively solves for $\langle Z\rangle$ and $n_{e}$ when everything else is fixed. We note that while argon results are discussed in this study, because of its prevalence in prior experimental studies, the general physics outcomes of runaway enhanced perturbations are applicable to other high $\mathrm{Z}$ impurities such as neon.

Runaway enhanced ionization at low $T_{e}$ : With the addition of a relativistic tail, and the inclusion of enhanced inelastic scattering by this relativistic electron tail, a clear increase in $\langle Z\rangle$ and broadening of the CSD is observed for the argon discharge at low $T_{e}$, exemplified by Fig. 3 for $T_{e}=2$ $\mathrm{eV}$. Here, we see the addition of relativistic electrons sampling non-enhanced ICS increases $\langle Z\rangle$ slightly, and then the addition of enhanced relativistic ICS to the model further increases $\langle Z\rangle$ and promotes CSD broadening. This is a direct result of the relativistic tail of the EEDF being able to sample an increasing ICS at higher energies. A wider spread of charge states shown in Fig. 3 will also lead to very different radiative cooling pathways, discussed in the next section. Contrasting the yellow line with blue and green lines in Fig. 3, one can also see that the complex excitation-ionization pathways enabled by QED enhancement of the cross sections can deplete the $\mathrm{Ar}^{+}$population.

Considering $\langle Z\rangle$ variation with $T_{e}$ Fig. 4 demonstrates a clear increase in $\langle Z\rangle$ at $T_{e}<50 \mathrm{eV}$, when compared to a Maxwellian EEDF typically used in decoupled CR modeling. For benchmarking purposes the result for a thermal plasma computed with the LANL CR modeling code, ATOMIC ${ }^{12}$, is included in Fig. 4, and agrees with the present thermal model.

Runaway-induced ion charge state effects: The runawayinduced ionization at low $T_{e}$, which is expected for the current quench phase, can impact disruption physics and its modeling in a number of ways. These are generally known as ion 


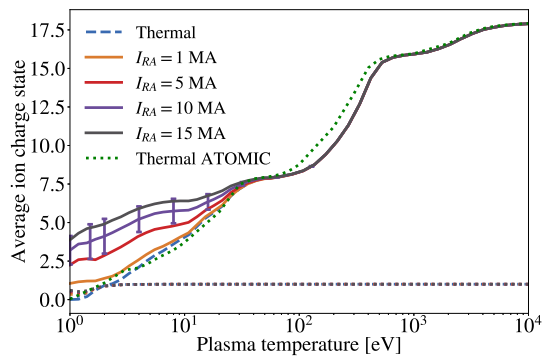

FIG. 4. Average argon charge state, $\langle Z\rangle$, over thermal $T_{e}$ demonstrating enhancement produced by relativistic electrons sampling enhanced relativistic ICS. $\langle Z\rangle$ for a thermal plasma computed by LANL ATOMIC code ${ }^{12}$ shown for benchmarking purposes. Average charge state of deuterium (...) shown for reference, indicating a fully stripped ion for nearly all plasma temperatures considered.

charge state effects, and here we give a few most prominent examples. The first is the enhanced pitch angle scattering of runaways due to the partial screening effect, which has a direct dependence on the ion charge state distribution, according to Hesslow et al. ${ }^{21,34}$. The physical importance is that enhanced pitch angle scattering would limit the energy of the $\mathrm{O}$-point of the runaway vortex, which is responsible for a lower runaway energy but broader pitch distribution ${ }^{35,36}$. The runaway-induced ionization would then modulate the pitch angle scattering rate due to partial screening, impacting both the avalanche threshold and runaway energy distribution, as well as the spatial transport of the runaways ${ }^{37}$.

The second example is the slowing down of the runaways by inelastic collisions with high-atomic-number impurities The runaway-induced ion charge state effect can be seen from the ICS for both excitation and ionization, Eqs. (5) and (10) Both ICS tend to decrease with higher ion charge number since the excitation energy $\Delta E_{i j}$ and ionization threshold energy $\Delta I_{Z}^{i}$ are generally higher with more electrons stripped from the atom. In the case of ionization ICS, the bound electron density $n_{e \alpha}$ would also decrease linearly with higher ion charge number. The impact of decreasing ICS with higher charge state is demonstrated by contrasting the inelastic Bethe stopping power in Eq. (1). In Fig. 5, we contrast the inelastic Bethe stopping power using (i) a CSD from the CR prediction of a background Maxwellian alone (ם), and (ii) CSD from the $\mathrm{CR}$ prediction that includes the runaway contribution $(\bullet)$. We find there is appreciable over-estimate of the inelastic Bethe stopping power if the runaway-induced corrections are not taken into account at low $T_{e}$, which is crucial in a post-thermal-quench plasma. At higher $T_{e}$, the curves converge as the CSD has converged between the two results. A these higher temperatures the inelastic losses fall as expected, at which point the well-known Coulomb drag of free electron elastic collisions becomes the dominant contribution to tota runaway drag.

The third example is more conventional, and it concerns the effect of Coulomb collisions on thermal bulk electrons. This

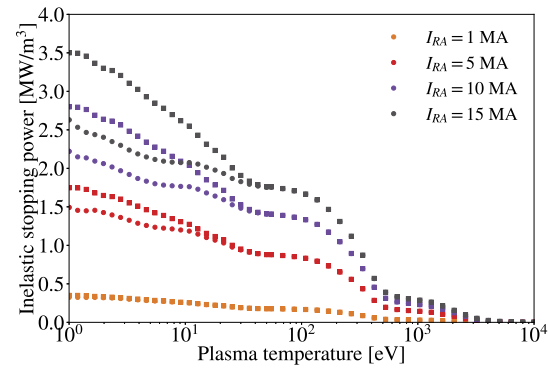

FIG. 5. Inelastic collision stopping power experienced by relativistic runaway electrons colliding with argon ions. Results shown contrast using a runaway-enhanced CSD $(\bullet)$ compared to a thermal plasma CSD (ם). Values computed with the Bethe stopping power formula Eq. (1).

comes from the strong $Z_{\mathrm{eff}}$ dependence for thermal plasma conductivity, and the collisional damping of both the externally injected ${ }^{38}$ and self-induced ${ }^{39}$ fast waves, by thermal electrons. The runaway-induced ionization, in the plasma regime of a mitigated tokamak disruption, can dramatically increase $Z_{\text {eff }}$, and hence increase dissipation of the remnant Ohmic current and the fast waves. The latter is particularly unfortunate since resonant wave-particle interaction offers a valuable tool for controlling the runaway energy ${ }^{38}$.

Runaway-induced radiative power loss (RPL) effects: While it is expected that radiative power loss, shown in Fig. 6, would be dominated by collisional excitation and ionization by runaways at low $T_{e}\left(T_{e}<4 \mathrm{eV}\right)$, by virtue of a higher charge state, there is also a subtler runaway effect when RPL rates by thermal electrons and runaways are comparable. This can be seen in Fig. 6, in the range of $5 \leq T_{e} \leq 100 \mathrm{eV}$, where the $\mathrm{RPL}$ of the combined thermal plus runaway plasma is up to $40 \%$ lower than that of the pure thermal plasma. The reduced RPL of the runaway enhanced discharges is found to be a result of the relativistic ICS enhancement smearing both the relative population of ion charge states and excited levels across a wider range, resulting in a smaller RPL in this temperature range. At higher temperatures, all RPL results effectively converge above approximately $100 \mathrm{eV}$, which is also seen in the result of the average charge state. Convergence occurs once the thermal population begins to dominate the ionization balance of the discharge, negating the excitation and ionization channels introduced by the relativistically enhanced scattering.

Uncertainty quantification: To validate the qualitative trends observed in this study, we employed UQ software Dakota $^{40}$ to perform an interval estimation uncertainty analysis by scaling the relativistic electron ICS to see the range of outcomes produced. In this UQ analysis, prefactors, $0.5 \leq$ $C_{E}, C_{I} \leq 2$, were applied to the relativistic excitation and ionization ICS formulas (5) and (10) to describe uncertainty in the approximate formulas used in this work. For $\langle Z\rangle$ and RPL appearing in Figs. 4 and 6, uncertainty bounds are shown. The 


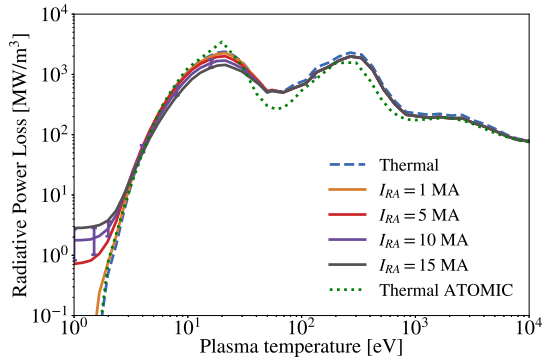

FIG. 6. RPL of argon over thermal $T_{e}$ demonstrating enhanced RPL produced by relativistic electrons sampling relativistic inelastic ICS RPL of thermal plasma computed with LANL ATOMIC code ${ }^{12}$ is shown for benchmarking purposes.

confidence intervals computed via 500 model evaluations with Latin hypercube sampling over the range of ICS uncertainty supports our observation that there is a definitive enhancement in both $\langle Z\rangle$ and total RPL of argon ions considered as a direct result of relativistic electrons sampling relativistically enhanced ICS.

Conclusion: A minority relativistic electron component, at a number density three orders of magnitude smaller than the low-temperature thermal bulk, can dominate the CSD in the presence of high-atomic-number impurities, due to the relativistic enhancement of the collisional excitation and ionization ICS. The resulting charge state effects can impact (1) radiative power loss rate and related Bethe stopping power on the runaways, and (2) collisional dissipation of Ohmic current and externally-injected and self-excited plasma waves. These physics findings suggest the necessity of a CR model that takes into account both the bulk electron population and the minority relativistic electron component, if high-atomicnumber impurities are present. With regard to actual tokamak experiments, especially on existing machines, the physics we have outlined here clarifies the conditions in which the runaways may or may not have a significant role in ionization balance. With a lower runaway density and higher electron temperature, the role of runaways in modifying the ionization and power balance is diminished (see Fig. 4 and 6). Similarly, the relativistic rise of the collisional cross section only occurs beyond a threshold energy of about $1 \mathrm{MeV}$ (see Fig. 1 and 2), so runaways of modest energy are unlikely to dominate ionization balance unless the background electron temperature becomes extremely low. Unpublished sources on major tokamak experiments appear to indicate experimental shots of two classes: ones with no identified elevated charge states and the other with elevated charge states that would dramatically upset the power balance estimate if ionization balance is solely attributed to a thermal background plasma. The physics detailed and the computational model developed here should allow specific calculations to help understand the underlying causes. There is also the possibility that experimental measurement of ionization balance, with the help of a computa- tional model reported here, can be used to impose an additional constraint on runaway density and energy.

This work was jointly supported by the U.S. Department of Energy through the Magnetic Fusion Theory Program and the Tokamak Disruption Simulation (TDS) SciDAC project at both Los Alamos National Laboratory (Contract No. 89233218CNA000001) and Sandia National Laboratory (Contract No. DE-NA-0003525). Additional support is provided by Laboratory Directed Research and Development program of Los Alamos National Laboratory under project number 20200356ER. T. Elder was also supported by the Science Undergraduate Laboratory Internships (SULI) program.

${ }^{1}$ M. Lehnen and S. Maruyama, Executive report of the Disruption Mitigation Workshop, Tech. Rep. ITR-18-002 (ITER Organization, St-Paul-lezDurance, 2018)

${ }^{2}$ M. Lehnen, K. Aleynikova, P. B. Aleynikov, D. J. Campbell, P. Drewelow, N. W. Eidietis, Y. Gasparyan, R. S. Granetz, Y. Gribov, N. Hartmann, E. M Hollmann, V. A. Izzo, S. Jachmich, S. H. Kim, M. Kočan, H. R. Koslowski, D. Kovalenko, U. Kruezi, A. Loarte, S. Maruyama, G. F. Matthews, P. B Parks, G. Pautasso, R. A. Pitts, C. Reux, V. Riccardo, R. Roccella, J. A. Snipes, A. J. Thornton, and P. C. de Vries, Journal of Nuclear Materials PLASMA-SURFACE INTERACTIONS 21, 463, 39 (2015).

D. G. Whyte, T. C. Jernigan, D. A. Humphreys, A. W. Hyatt, C. J. Lasnier, P. B. Parks, T. E. Evans, P. L. Taylor, A. G. Kellman, D. S. Gray, and E. M. Hollmann, Journal of Nuclear Materials Plasma-Surface Interactions in Controlled Fusion Devices 15, 313-316, 1239 (2003).

${ }^{4}$ R. Granetz, D. G. Whyte, V. A. Izzo, T. Biewer, M. L. Reinke, J. Terry, R. Granetz, D. G. Whyte, V. A. Izzo, T. Biewer, M. L. Reinke, J. Terry,
A. Bader, M. Bakhtiari, T. Jernigan, and G. Wurden, Nuclear Fusion 46 , A. Bader, M.

${ }^{5}$ E. M. Hollmann, M. E. Austin, J. A. Boedo, N. H. Brooks, N. Commaux, N. W. Eidietis, D. A. Humphreys, V. A. Izzo, A. N. James, T. C. Jernigan, A. Loarte, J. Martin-Solis, R. A. Moyer, J. M. Muñoz-Burgos, P. B. Parks, D. L. Rudakov, E. J. Strait, C. Tsui, M. A. V. Zeeland, J. C. Wesley, and J. H. Yu, Nuclear Fusion 53, 083004 (2013).

${ }^{6}$ C. Paz-Soldan, N. W. Eidietis, R. Granetz, E. M. Hollmann, R. A. Moyer, J. C. Wesley, J. Zhang, M. E. Austin, N. A. Crocker, A. Wingen, and Y. Zhu, Physics of Plasmas 21, 022514 (2014)

${ }^{7}$ A. H. Boozer, Physics of Plasmas 22, 032504 (2015).

${ }^{8}$ A. V. Gurevich, G. M. Milikh, and R. Roussel-Dupre, Physics Letters A 165, 463 (1992).

${ }^{9}$ L. Fletcher, B. R. Dennis, H. S. Hudson, S. Krucker, K. Phillips, A. Veronig, M. Battaglia, L. Bone, A. Caspi, Q. Chen, P. Gallagher, P. T. Grigis, H. Ji, W. Liu, R. O. Milligan, and M. Temmer, Space Science Reviews 159, 19 (2011)

${ }^{10} \mathrm{G}$. Israelian and G. Meynet, eds., The Metal Rich Universe, (Cambridge University Press, Cambridge, UK, 2008).

${ }^{11}$ X. Llovet, C. J. Powell, F. Salvat, and A. Jablonski, Journal of Physical and Chemical Reference Data 43, 013102 (2014).

${ }^{12}$ C. J. Fontes, H. L. Zhang, J. A. Jr, R. E. H. Clark, D. P. Kilcrease, J. Colgan, R. T. Cunningham, P. Hakel, N. H. Magee, and M. E. Sherrill, J. Phys. B: At. Mol. Opt. Phys. 48, 144014 (2015)

${ }^{13}$ J. H. Scofield, Physical Review A 18, 963 (1978)

${ }^{14}$ C. J. Bostock, C. J. Fontes, D. V. Fursa, H. L. Zhang, and I. Bray, Phys. Rev. A 88, 012711 (2013)

${ }^{15}$ C. J. Fontes, C. J. Bostock, and K. Bartschat, EPJ H 39, 517 (2014),

${ }^{16} \mathrm{H}$. Bethe, Z. Physik 76, 293 (1932)

${ }^{17}$ M. Inokuti, Rev. Mod. Phys. 43, 297 (1971)

${ }^{18}$ V. Berestetskii, E. Lifshitz, and L. Pitaevskii, Quantum Electrodynamics: Volume 4 (Course of Theoretical Physics) (Butterworth-Heinemann, 1982). ${ }^{19}$ B. N. Breizman, P. Aleynikov, E. M. Hollmann, and M. Lehnen, Nuclear Fusion 59, 083001 (2019).

${ }^{20}$ L. Hesslow, O. Embréus, M. Hoppe, T. C. DuBois, G. Papp, M. Rahm, and T. Fülöp, Journal of Plasma Physics 84 (2018), M. Rahm, and T. Fülö,

${ }^{21}$ L. Hesslow, O. Embréus, A. Stahl, T. DuBois, G. Papp, S. Newton, and T. Fülöp, Physical Review Letters 118, 255001 (2017)

${ }^{22}$ E. M. Hollmann, N. W. Eidietis, J. L. Herfindal, P. B. Parks, A. Y. Pigarov, 
D. Shiraki, M. E. Austin, L. Bardoczi, L. Baylor, I. Bykov, T. N. Carlstrom, D. Kaplan, C. J. Lasnier, A. Lvovskiy, A. Moser, R. A. Moyer, C. Paz Soldan, D. L. Rudakov, C. Samuell, M. Shafer, M. V. Zeeland, A. Welander, and R. Wilcox, Nuclear Fusion 59, 106014 (2019).

${ }^{23}$ H. K. Chung, M. H. Chen, W. L. Morgan, Y. Ralchenko, and R. W. Lee,

High Energy Density Physics 1, 3 (2005).
${ }^{24}$ H. K. Chung, C. Bowen, C. J. Fontes, S. B. Hansen, and Y. Ralchenko,

${ }^{24}$ H. K. Chung, C. Bowen, C. J. Fontes, S. B.
High Energy Density Physics 9, 645 (2013).

${ }^{5}$ R. Piron, F. Gilleron, Y. Aglitskiy, H. K. Chung, C. J. Fontes, S. B. Hansen, O. Marchuk, H. A. Scott, E. Stambulchik, and Y. Ralchenko, High Energy Density Physics 23, 38 (2017)

${ }^{26}$ J. Bretagne, G. Callede, M. Legentil, and V. Puech, J. Phys. D: Appl. Phys. 19, 761 (1986).

${ }^{27}$ H. van Regemorter, The Astrophysical Journal 136, 906 (1962).

${ }^{28}$ H. K. Chung, R. W. Lee, and M. H. Chen, High Energy Density Physics 3 , 342 (2007).

${ }^{29}$ Y.-K. Kim, Phys. Rev. A 64, 032713 (2001).

${ }^{30}$ C. Møller, Annalen der Physik 406, 531 (1932).

${ }^{31} \mathrm{~A}$. Burgess and M. C. Chidichimo, Monthly Notices of the Royal Astronomical Society 203, 1269 (1983).

${ }^{32}$ H. Bethe, F. Hund, N. F. Mott, W. Pauli, A. Rubinowicz, G. Wentzel, and A. Smekal, Quantentheorie, 2nd ed., Handbuch der Physik (SpringerVerlag, Berlin Heidelberg, 1933)

${ }^{33} \mathrm{X}$. J. Wang, J. Seuntjens, and J. M. Fernández-Varea, Journal of Physics B: Atomic, Molecular and Optical Physics 51, 145201 (2018).

${ }^{34}$ L. Hesslow, O. Embréus, G. J. Wilkie, G. Papp, and T. Fülöp, Plasma Physics and Controlled Fusion 60, 074010 (2018)

${ }^{35}$ C. J. McDevitt, Z. Guo, and X.-Z. Tang, Plasma Physics and Controlled Fusion 60, 024004 (2018)

${ }^{36}$ Z. Guo, C. J. McDevitt, and X.-Z. Tang, Plasma Physics and Controlled Fusion 59, 044003 (2017)

${ }^{37}$ C. J. McDevitt, Z. Guo, and X.-Z. Tang, Plasma Physics and Controlled Fusion 61, 024004 (2019).

${ }^{38}$ Z. Guo, C. J. McDevitt, and X.-Z. Tang, Physics of Plasmas 25, 032504 (2018).

${ }^{39}$ C. Liu, E. Hirvijoki, G.-Y. Fu, D. P. Brennan, A. Bhattacharjee, and C. PazSoldan, Physical Review Letters 120, 265001 (2018).

${ }^{40}$ B. Adams, M. Ebeida, M. Eldred, G. Geraci, J. Jakeman, K. Maupin, J. Monschke, J.A. Stephens, L. Swiler, D. Vigil et al., Dakota: A Multilevel Parallel Object-Oriented Framework for Design Optimization, Parameter Estimation, Uncertainty Quantification, and Sensitivity Analysis: Version Estimation,
6.7 (2017). 


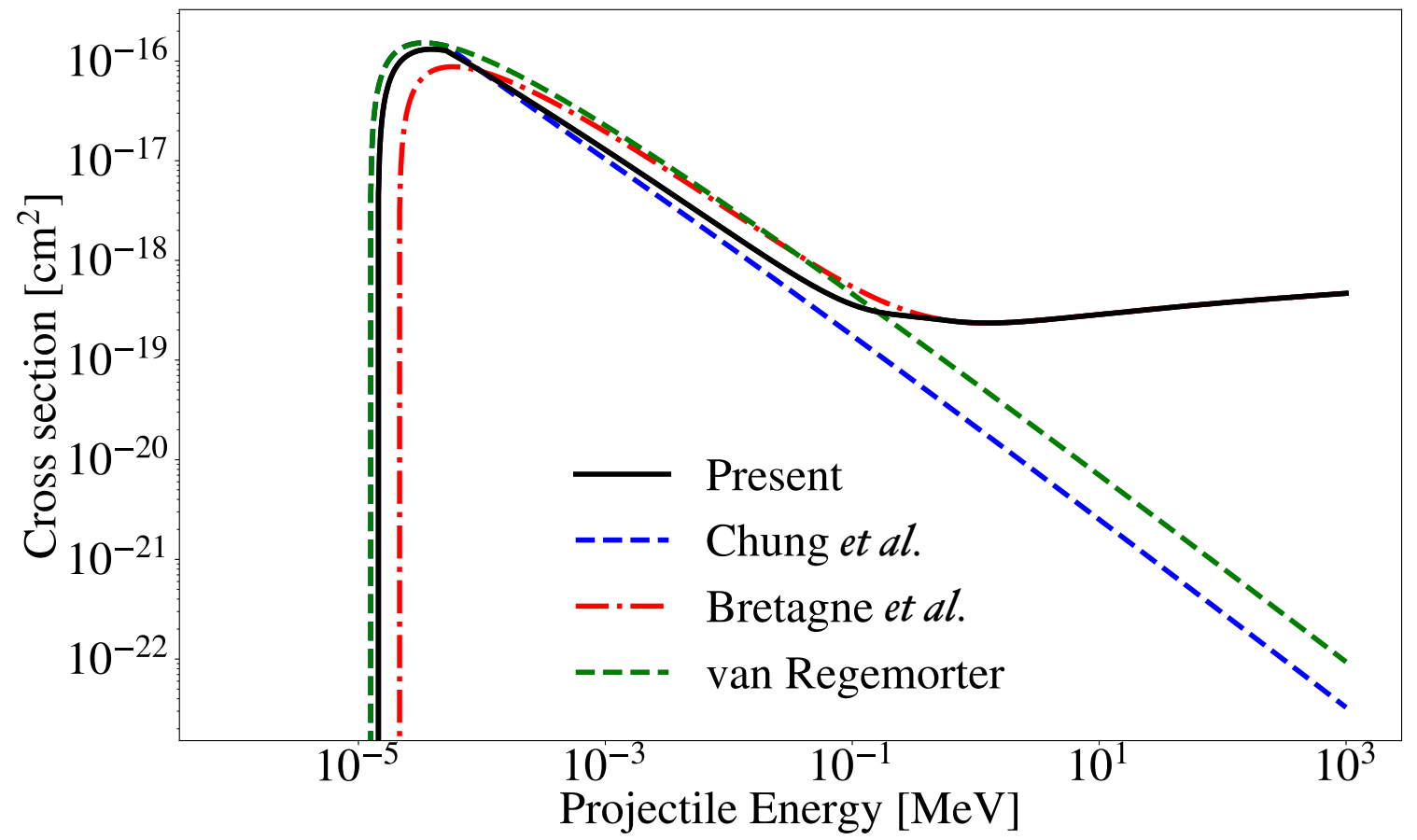




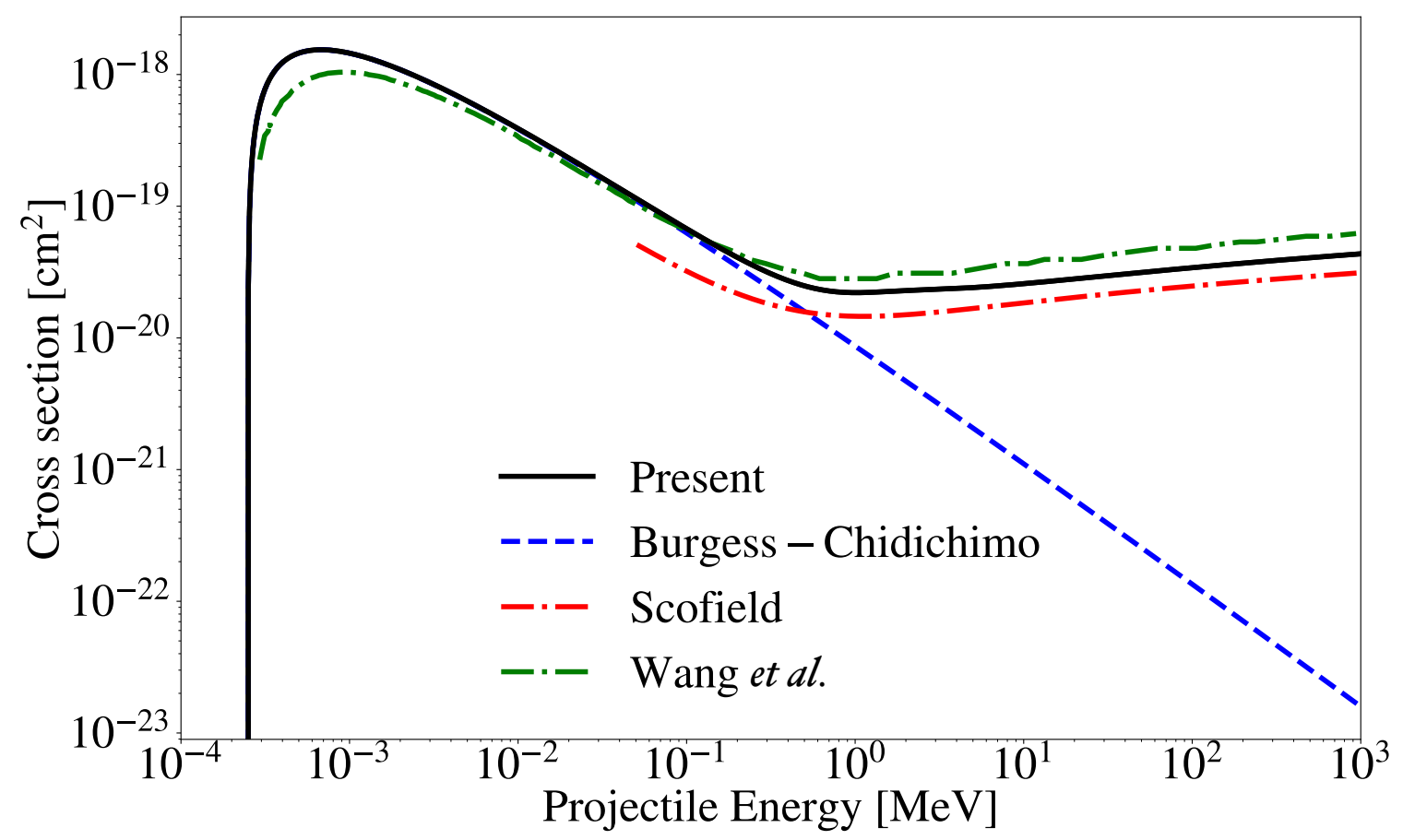




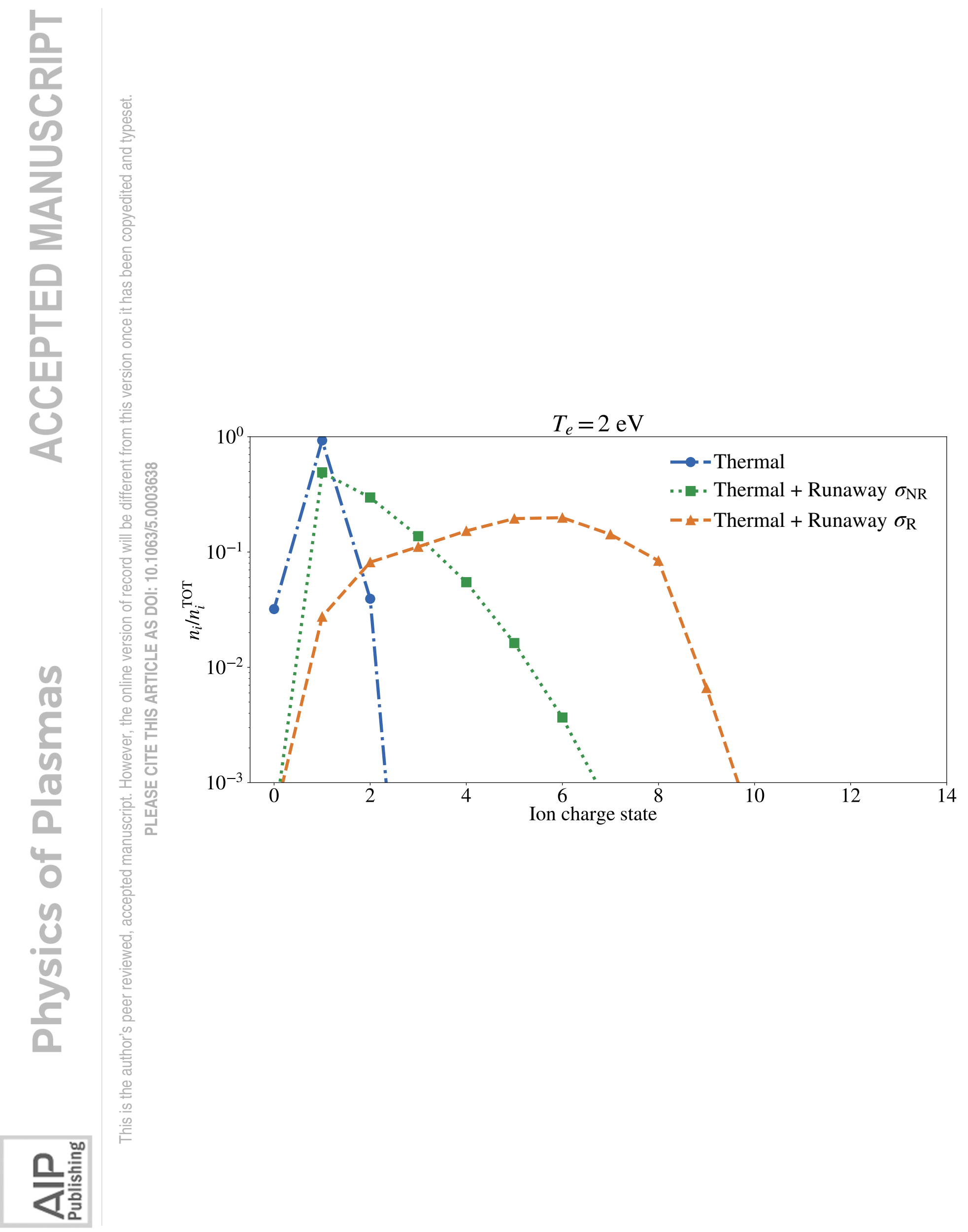




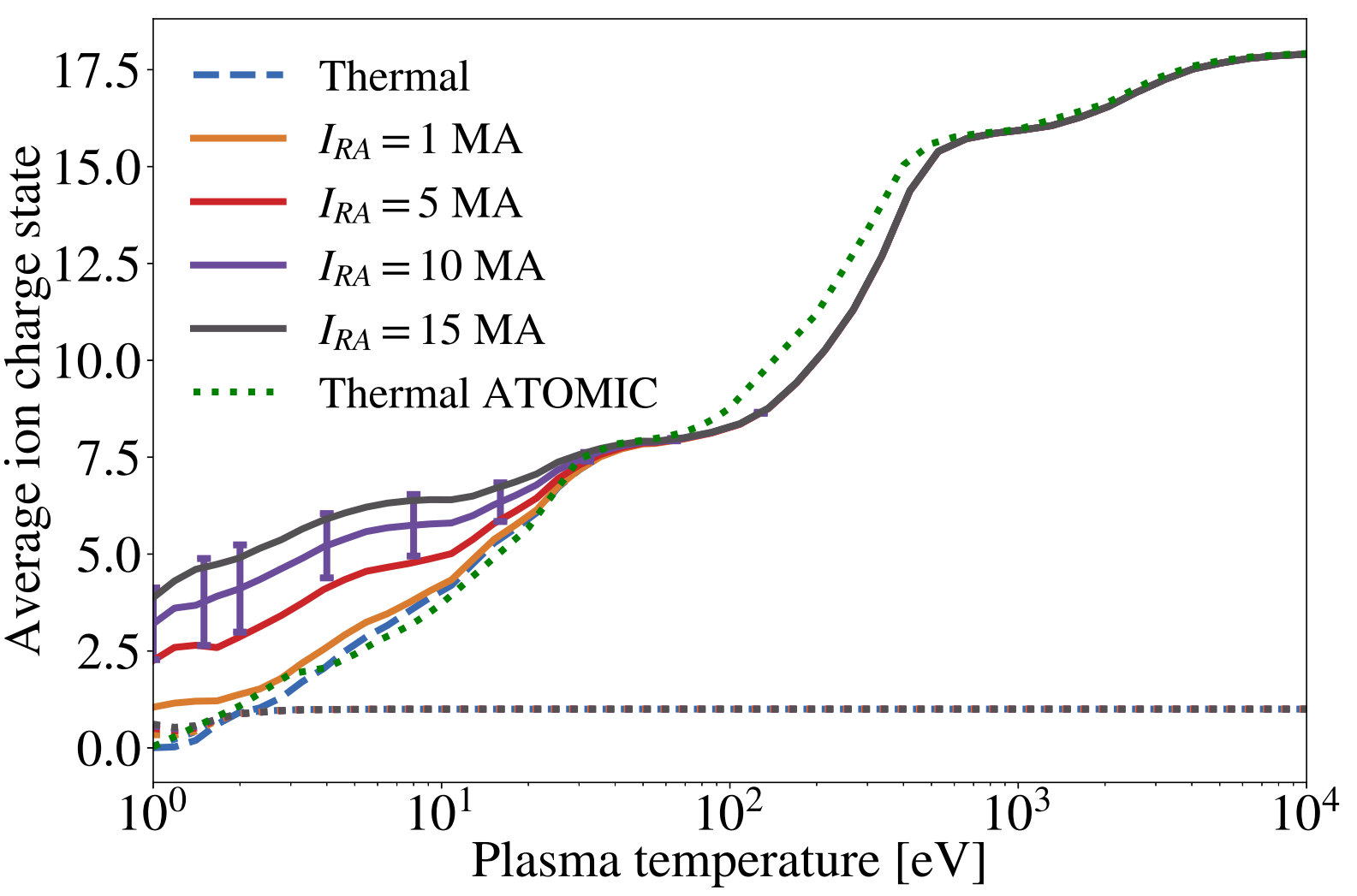




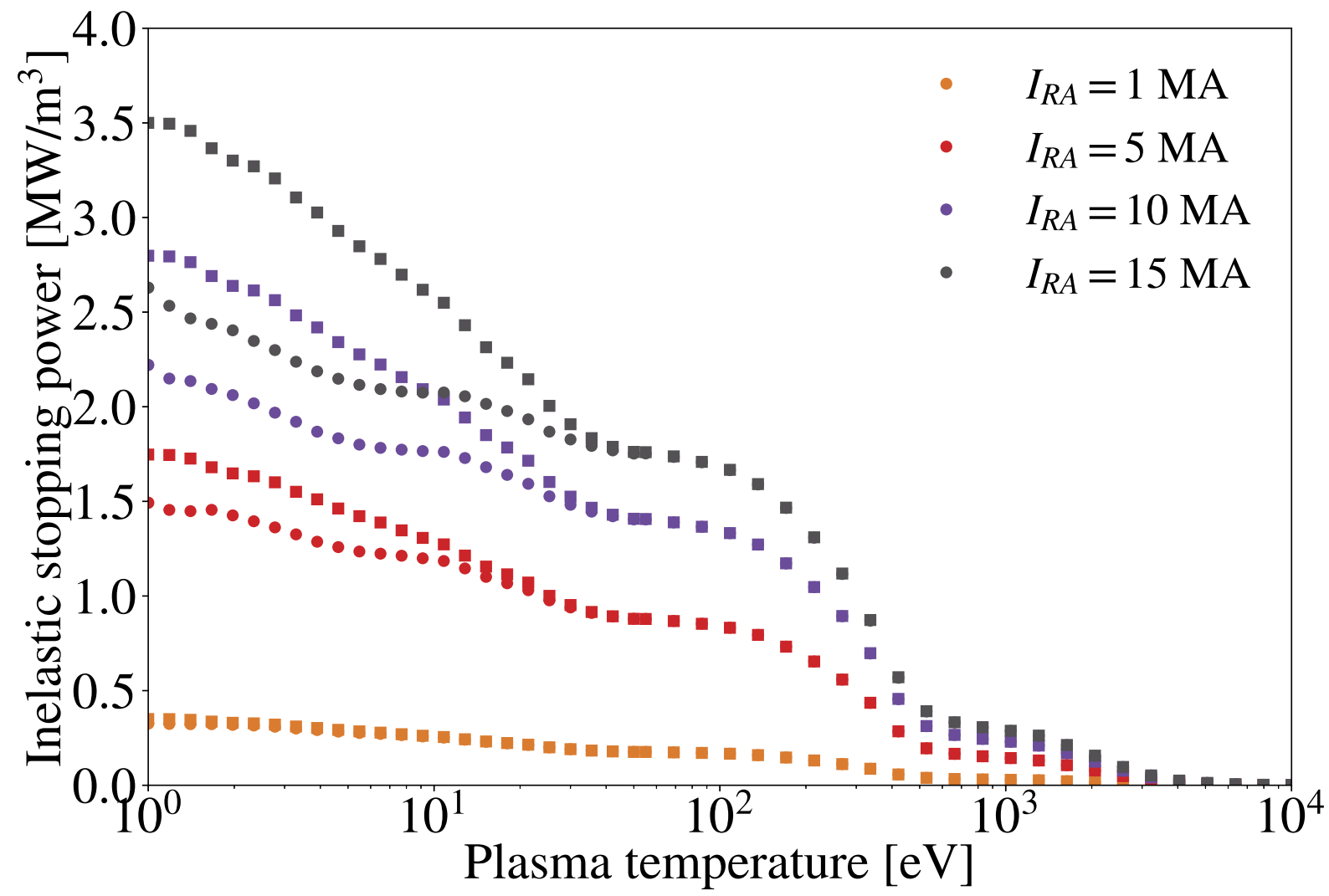




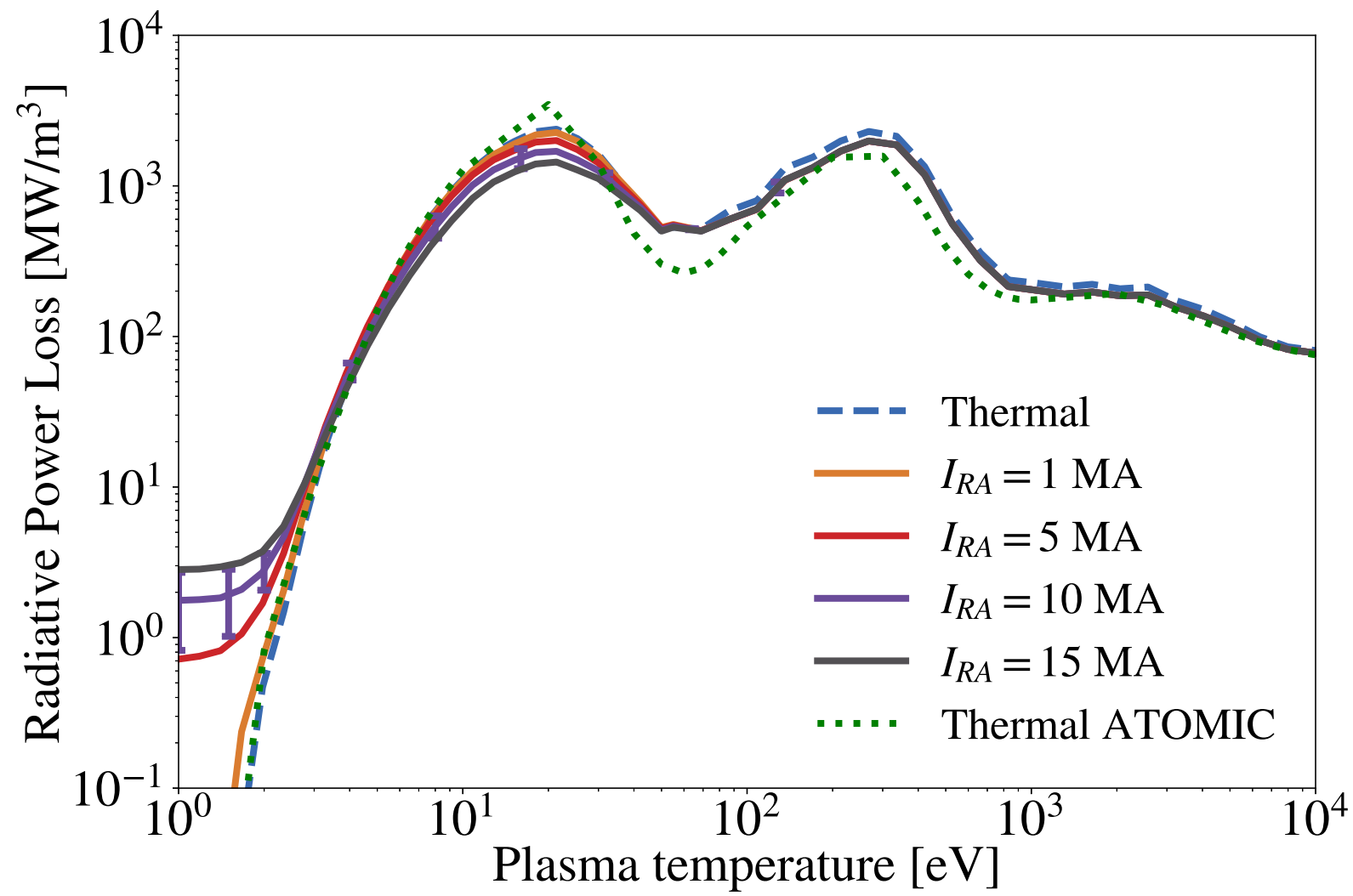

